\title{
Rapid and Reversible Astrocytic Reaction to Afferent Activity Blockade in Chick Cochlear Nucleus
}

\author{
Karen S. Canady and Edwin W Rubel \\ Hearing Development Laboratories, University of Washington, Seattle, Washington 98195
}

\begin{abstract}
We describe here rapid proliferation of astrocytic processes immunoreactive for glial fibrillary acidic protein (GFAP) in the chick cochlear nucleus following blockade of action potentials in the afferent nerve. Unilateral eighth nerve activity blockade was achieved through intralabyrinthine injection of TTX. Within 1 hr of activity blockade, a $56 \%$ increase in area density of GFAP-immunoreactive processes was found in the ipsilateral cochlear nucleus as compared to the contralateral side of the brain. This increase reached $152 \%$ by 3 hr. When eighth nerve activity was allowed to recover and animals were studied 1 week after TTX injection, no difference was found in GFAP immunoreactivity between the ipsilateral and contralateral cochlear nuclei. This is the first report of a glial reaction to documented neuronal inactivity in the absence of neuropathology. These results indicate that neuronal activity may regulate the structure of astrocytic processes.
\end{abstract}

Numerous accounts of structural changes in glial cells occurring in regions of neuronal injury and degeneration describe an increase in astrocytic immunoreactivity for glial fibrillary acidic protein (GFAP) and an increase in astrocytic process length and number (Eng and DeArmond, 1982; Lindsay, 1986; Malhotra et al., 1990). The experimental manipulations causing neuronal damage and degeneration accompanied by such astrocytic reactions include axotomy (Graeber and Kreutzberg, 1988), freezing lesions (Amaducci et al., 1981), stab wounds (Miyake et al., 1988), and deafferentation (Wong-Riley, 1972; Cass and Goshgarian, 1990). Increased GFAP immunoreactivity following such lesions first appears 1-10 d after injury, with the exception of a freezing lesion of rat cortex, which resulted in increased GFAP immunoreactivity within $30 \mathrm{~min}$ (Amaducci et al., 1981). Some investigators have hypothesized that these glial reactions are caused by degeneration products and result in a permanent scar that impedes neuronal recovery (Reier, 1986).

We have previously reported a rapid increase in GFAP-immunoreactive (GFAP+) and silver-impregnated glial processes in the chick cochlear nucleus, nucleus magnocellularis (NM), following removal of the cochlea (Rubel et al., 1990; Rubel and MacDonald, 1992). These increases are seen within $1 \mathrm{hr}$ of

\footnotetext{
Received July 17, 1991; revised Oct. 15, 1991; accepted Oct. 18, 1991.

This work was supported by USPHS Grant DC00393 and has been presented previously in abstract form. We thank JiaLin Shang for measuring neuron size and number, and Jack Winterowd for providing and preparing calcium alginate. Richard L. Hyson, Ed Lachica, and Diana Lurie contributed helpful comments on an earlier version of the manuscript.

Correspondence should be addressed to Edwin W Rubel, Hearing Development Laboratories, RL-30, University of Washington, Seattle, WA 98195.

Copyright (C) 1992 Society for Neuroscience $0270-6474 / 92 / 121001-09 \$ 05.00 / 0$
}

cochlea removal and are dramatic by $6 \mathrm{hr}$, long before signs of eighth nerve terminal degeneration are seen (Parks and Rubel, 1978; Parks, 1981). The rapidity of this glial reaction led us to hypothesize that the signal initiating the glial changes is the cessation of neuronal action potentials rather than signals specific to neuronal degeneration.

NM neurons in the chick receive their only excitatory input from the ipsilateral basilar papilla (avian cochlea) via eighth nerve fibers (Fig. 1). Thus, either removal of the basilar papilla or perilymphatic injection of TTX is sufficient to eliminate all extracellularly recorded action potentials in NM (Born and Rubel, 1988; Born et al., 1991). This unilateral manipulation leaves neuronal activity in the contralateral NM unaltered and provides a within-animal and within-tissue section control.

In this study, eighth nerve activity blockade was achieved by injection of TTX into the perilymph bathing the otolithic organs. This perilymph is continuous with that which bathes the peripheral processes of auditory nerve axons in the cochlea. Thus, the TTX had access to voltage-dependent sodium channels on auditory nerve axons. Since these axons provide the only excitatory input to NM, blocking eighth nerve activity prevents spike activity in NM neurons. Successful blockade and the restoration of normal function were monitored by recording evoked potential amplitude and threshold from the brainstem. We report here that cessation of neuronal action potentials is sufficient to induce a rapid increase in GFAP+ astrocytic processes in the ipsilateral NM. This astrocytic reaction is reversed following the resumption of eighth nerve activity.

\section{Materials and Methods}

Subjects

The subjects for all experiments were 1-2-week-old white Leghorn chickens. Eggs were obtained from a local supplier (H\&N, Redmond, WA) and incubated and hatched in the University of Washington vivarium in AAALAC-approved facilities. Animals were given free access to food and water at all times. The numbers of chickens used for each condition are given in the Results.

\section{Short-term activity blockade}

Tetrodotoxin. TTX was embedded in either polyvinyl alcohol (PVA; DuPont) or calcium alginate to enable slow release. PVA $(16 \% \mathrm{w} / \mathrm{v})$ was dissolved in distilled water warmed to $80^{\circ} \mathrm{C}$. Once the PVA solution cooled to room temperature, $20 \mu \mathrm{l}$ of $3 \mathrm{mM}$ TTX (20 $\mu \mathrm{g}$; Sigma, St. Louis, MO) in citrate buffer were added to $200 \mu \mathrm{l}$ of the $16 \%$ PVA solution. The TTX/PVA solution was allowed to sit overnight and was used within $24 \mathrm{hr}$ as the PVA solution hardens with time at room temperature.

The calcium alginate (with and without TTX) was provided by Jack Winterowd of the Department of Polymer Chemistry, College of Forest Resources, University of Washington. The calcium alginate was in spherical form. Individual beads averaged $500 \mu \mathrm{m}$ in diameter and 


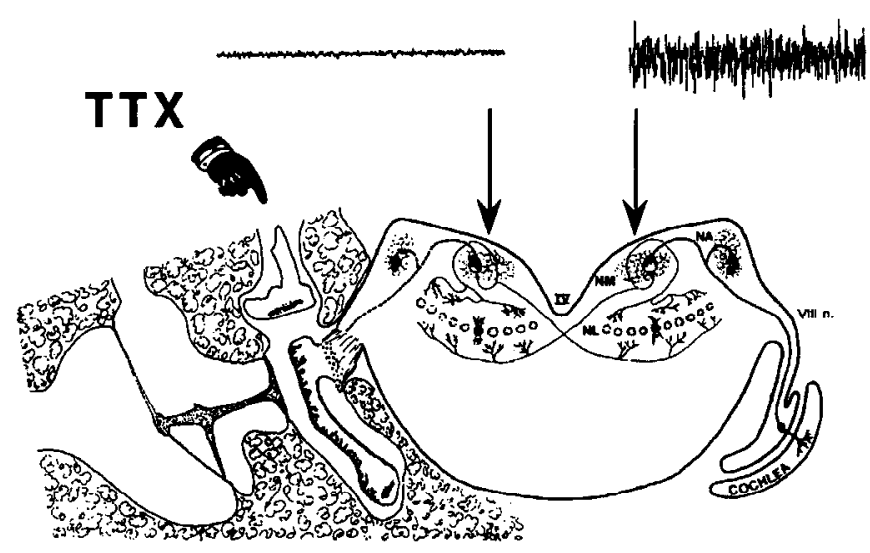

Figure 1. Schematic diagram of chick brainstem auditory nuclei. Axons of the eighth nerve $(V I I I n$. $)$ project ipsilaterally to nucleus angularis $(N A)$ and NM. NM neurons project bilaterally to nucleus laminaris $(N L)$. All extracellularly recorded spike activity in NM is eliminated by cochlea removal or by intralabyrinthine TTX injection (Born et al., 1991).

contained approximately $250 \mathrm{ng}$ TTX. The calcium alginate/TTX beads were stored at $-70^{\circ} \mathrm{C}$ for up to 6 months.

Surgical procedures. Chicks were deeply anesthetized with a combination of ketamine $(80 \mathrm{mg} / \mathrm{kg}$ body weight) and sodium pentobarbital ( $15 \mathrm{mg} / \mathrm{kg}$ body weight). A $1 \mathrm{~cm}$ incision was made originating $2 \mathrm{~mm}$ posterior to the ear canal and oriented toward the vertex. A region of spongy bone was removed, revealing the smooth, thin bone overlying the vestibule. The bent tip of a 23 gauge needle was used to puncture the bone over the saccule and create a hole $0.5 \mathrm{~mm}$ in diameter. TTX was inserted through this hole in one of two forms: (1) as two calcium alginate/TTX beads (500 ng TTX total) or (2) as a slurry of approximately $10 \mu \mathrm{l}$ PVA with $1 \mu \mathrm{g}$ TTX, which was injected into the perilymph through a 23 gauge stub adaptor. Control animals received one of the following: calcium alginate, PVA, saline, surgery only, or no surgery.

Auditory evoked potentials. Born and Rubel (1988) have demonstrated that far-field evoked potentials to acoustic stimuli [auditory brainstem responses (ABRs)] disappear following TTX injection into the perilymph in association with the loss of spikes recorded extracellularly with a microelectrode in NM. In the present study, the less invasive approach of recording ABRs was used to evaluate the effectiveness and duration of eighth nerve activity blockade after treatment with TTX embedded in PVA or calcium alginate. ABRs were recorded using subdermal pin electrodes placed in the comb, vertex, and thigh (ground). The stimulus was a 2 msec broad-band click with a maximal intensity of $60 \mathrm{~dB}$ peak equivalent sound pressure level presented as an alternating square wave through a Realistic Minimus-7 speaker. Responses were amplified, filtered to pass $30-3000 \mathrm{~Hz}$, digitized by an analog-to-digital converter at a rate of $10 \mathrm{kHz}$, and then averaged over 250 stimulus presentations. Amplitude was defined as the difference (in $\mu \mathrm{V}$ ) between the most positive and most negative peaks of the ABR waveform.

Evoked potential amplitude and threshold were determined (1) prior to puncture of the vestibule, (2) following the puncture but prior to introduction of TTX or vehicle, and (3) at $5 \mathrm{~min}$ intervals beginning immediately after TTX introduction to the perilymph until a response could no longer be elicited by $60 \mathrm{~dB}$ clicks (or for $20 \mathrm{~min}$ in the case of vehicle injection). Animals were reanesthetized after 3-8 hr to determine the duration of eighth nerve activity blockade and after $1-4 \mathrm{~d}$ to document the recovery of amplitude and threshold of the ABR.

In order to confirm that this method of intralabyrinthine TTX treatment was sufficient to block ipsilateral auditory nerve activity completely, the contribution of the contralateral auditory pathway to the ABR was eliminated by removal of the contralateral cochlea. In these animals the criterion for successful activity blockade was complete elimination of ABRs to $60 \mathrm{~dB}$ clicks. Because the contralateral cochlea removal would result in increased GFAP immunoreactivity in NM, the contralateral NM could not be used as a within-animal control for studying GFAP immunoreactivity after ipsilateral TTX injection. Therefore, these chicks were not perfused for histology.

Immunocytochemistry. At 1, 3, or $6 \mathrm{hr}$ after placement of TTX or vehicle in the perilymph, chicks were reanesthetized and perfused trans- cardially with $0.9 \%$ saline for 1-2 min followed by $4 \%$ paraformaldehyde in phosphate buffer for 10-15 min. The brains were removed, blocked, and then immersed in $4 \%$ paraformaldehyde for 12-18 hr. Afterward, the brains were placed in either PBS for $3-6 \mathrm{hr}$ or $30 \%$ sucrose in PBS overnight. Vibratome sections were cut $30 \mu \mathrm{m}$ thick from blocks stored in PBS. Individual sections, freely floating in $30 \%$ sucrose in PBS, were allowed to equilibrate, frozen on dry ice for $10 \mathrm{~min}$, and then thawed slowly at $4^{\circ} \mathrm{C}$. The remaining brains, after sinking in 30\% sucrose, were frozen and sectioned at $10-20 \mu \mathrm{m}$ using a cryostat at $-25^{\circ} \mathrm{C}$ and thaw mounted on chrome-alum-subbed glass slides.

Cryostat sections were rehydrated in a graded series of ethanols followed by PBS. The tissue sections (thaw mounted or free floating) were then incubated in $4 \%$ normal goat serum for $1 \mathrm{hr}$. This and all other immunocytochemical reagents were prepared in $1 \% \mathrm{BSA}, 0.1 \%$ sodium azide (except the $A B C$ reagent) in PBS. The sections were then incubated in rabbit serum immunized against bovine GFAP (DAKO, Santa Barbara, CA). Cryostat sections were incubated in primary antiserum diluted $1: 400$ to $1: 2400$ overnight in a humidified chamber at room temperature. Free-floating vibratome sections were incubated in primary antiserum diluted 1:1200 to 1:9600 for $2 \mathrm{~d}$ at room temperature on a shaker table. Control sections were incubated in normal rabbit IgG diluted 1:10,000. Following the incubation in primary antiserum and all subsequent reagents, the sections underwent three $10 \mathrm{~min}$ washes in PBS. The sections were then incubated in biotinylated goat anti-rabbit serum, diluted 1:200, for $2 \mathrm{hr}$, washed, and then incubated in an avidinbiotin complex (Vectastain ABC Kit, Vector Labs, Burlingame, CA). Diaminobenzidine $(0.25 \mathrm{mg} / \mathrm{ml}$; Sigma, St. Louis, MO) with $0.1 \%$ hy-

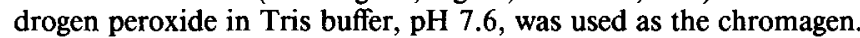

Data analysis. The relative intensity of GFAP immunolabeling in NM ipsilateral and contralateral to activity blockade was analyzed using a BioQuant Image Analysis system (R\&M Biometrics, Nashville, TN). At least three sections per animal were analyzed using a $40 \times$ objective on a Leitz Aristoplan microscope. The microscopic image was relayed by a Dage MTI 68 videocamera to a video monitor. Sections were selected for measurement if they appeared evenly stained, were symmetric in the transverse plane, and contained sufficiently central portions of NM that three nonoverlapping measurements could be taken on each side. For each side of each section, one measurement was taken along the medial border of NM, one along the lateral border, and one in the center. A digitizing tablet was used to designate the area to be analyzed. The microdensitometry system was set up to scan the selected area of the digitized image and recognize pixels of the image whose optical density exceeded a predetermined threshold. The threshold was selected to detect intensely stained astrocytic processes. The same threshold as well as the same microscope and video camera settings were used for both sides of a given tissue section and usually for all sections on a given slide. The percentage of the selected area containing threshold pixels was then calculated for each of the three selected areas on each side of a tissue section. The three values for each side were then averaged, and a ratio of average percentage of threshold pixels for the side ipsilateral to TTX treatment versus the average percentage for the contralatcral side was determincd. Thus, for cach section, a percentage difference score was calculated such that a positive value reflects more GFAP+ astrocytic processes ipsilateral to TTX treatment.

\section{Recovery following activity blockade}

To ensure rapid recovery of eighth nerve activity following short-term blockade, TTX was diluted in saline rather than in a slow-release compound. A $10 \mu \mathrm{l}$ Hamilton syringe was used to inject $1-2 \mu \mathrm{l}$ of $0.3 \mathrm{~mm}$ TTX into the vestibule. Chicks $8-10$-d-old were anesthetized and prepared as described for the short-term experiments. Three separate groups of chicks were used for this study. The first group was used to determine the sufficiency of $0.3 \mathrm{~mm}$ TTX in saline to eliminate auditory evoked potentials. These chicks had their contralateral cochleae removed and their ABRs recorded before and after ipsilateral TTX injection. The second group of chicks was used to characterize the reduction in ABR amplitude that occurs when the contribution from only one ear has been eliminated. In these chicks, ABRs were recorded before and after unilateral cochlea removal.

The third group of chicks was the experimental group in which GFAP immunoreactivity was examined. These chicks underwent a short period of activity blockade followed by recovery of eighth nerve activity. For these animals, ABRs were recorded from the same subjects that would be used for histological analysis so that elimination and return of eighth nerve activity could be confirmed. Thus, in the present group of chicks, 
the contralateral cochlea remained intact and reduction (rather than elimination) of ABR amplitude was used to indicate successful unilateral activity blockade. ABRs were recorded again at $3 \mathrm{hr}$ after TTX injection to confirm a duration of activity blockade sufficient to result in a reliable glial reaction. Thereafter, ABRs were recorded no more than once daily to monitor recovery of $A B R$ amplitude. These animals were perfused 1 week after TTX injection. Only animals from this third group were studied histologically.

The brains of these chicks were prepared and immunocytochemically stained as described above, except that alternate tissue sections were stained with thionin for analysis of neuronal size and number in NM. This was done as a second means of confirming recovery from activity blockade and to allow for comparison of neuronal recovery to glial recovery. Neurons were counted from a one-in-two series of $20 \mu \mathrm{m}$ cryostat sections or from a one-in-four series of $30 \mu \mathrm{m}$ vibratome sections as described by Born and Rubel (1985). Only neurons with a stained cytoplasm, clear nuclear area, and a nucleolus were counted. Cross-sectional areas of neuronal somata were measured as previously described (Born and Rubel, 1985) at $30 \%$ and $70 \%$ of the total posteriorto-anterior length of NM. Fifty to $70 \mathrm{NM}$ neurons were measured on each side of the brain and at both levels of the anterior-posterior axis.

\section{Results}

Auditory evoked potentials

Figure 2 shows elimination and recovery of the ABR for one animal following treatment with TTX in PVA. TTX in PVA abolished the ABR to $60 \mathrm{~dB}$ clicks within 1-20 min in all 13 chicks receiving $1 \mu \mathrm{g}$ TTX. Responses returned after $6-9 \mathrm{hr}$ in three chicks and after $24-48 \mathrm{hr}$ in the remaining 10 animals. ABRs recovered to pre-TTX amplitude and threshold within 5 $\mathrm{d}$ in all but three chicks. PVA alone did not affect ABR amplitude or threshold $(n=5)$.

TTX in calcium alginate abolished the ABR to $60 \mathrm{~dB}$ clicks within 1-30 min in 9 of 11 chicks receiving approximatcly 500 ng TTX. Responses returned after $12-48 \mathrm{hr}$ and eventually recovered to pre-TTX amplitude and threshold in all affected animals. Calcium alginate alone was sufficient to reduce or eliminate the ABR to $60 \mathrm{~dB}$ clicks $(n=2)$. Presumably the effect of calcium alginate alone is a result of calcium released by the alginate as it breaks down. The ABR, however, always returned to normal at some point after calcium alginate treatment. Thus, neither the calcium alginate nor the TTX caused permanent damage to peripheral auditory structures.

In all 19 animals who underwent contralateral cochlea removal, $0.3 \mathrm{~mm}$ TTX in saline abolished the ABR to $60 \mathrm{~dB}$ clicks within $25 \mathrm{~min}$. Three chicks died within $2 \mathrm{hr}$ of the TTX injection. Five recovered near-normal ABR amplitude within 3$8 \mathrm{hr}$. The 11 remaining chicks had no ABR to $60 \mathrm{~dB}$ clicks for at least $3 \mathrm{hr}$ following TTX injection. Seven of these 11 chicks showed partial recovery of ABR amplitude within $12-48 \mathrm{hr}$. The remainder did not survive reanesthetization. Thus, $0.3 \mathrm{~mm}$ TTX delivered in saline is sufficient to eliminate auditory evoked potentials completely, but individual chicks must be monitored to confirm a minimum of $3 \mathrm{hr}$ of activity blockade.

For animals whose contralateral cochleae were left intact, ABR amplitude decreased by approximately $50 \%$ following either ipsilateral TTX injection or ipsilateral cochlea removal. With both cochleae intact, the amplitude of the ABR to $60 \mathrm{~dB}$ clicks ranged from 4.6 to $12.7 \mu \mathrm{V}(n=27)$. Following unilateral cochlea removal, the ABR amplitude averaged $50.3 \%$ of normal (range, $35-63 \% ; n=7$ ). Fifteen to $30 \mathrm{~min}$ following an intralabyrinthine injection of 1-2 $\mu$ l of $0.3 \mathrm{~mm}$ TTX, the amplitude averaged $49.1 \%$ of normal (range, $29-69 \%$; $n=18$ ). This excludes two animals whose ABR amplitudes were not affected by the TTX injection. ABR amplitudes remained reduced at

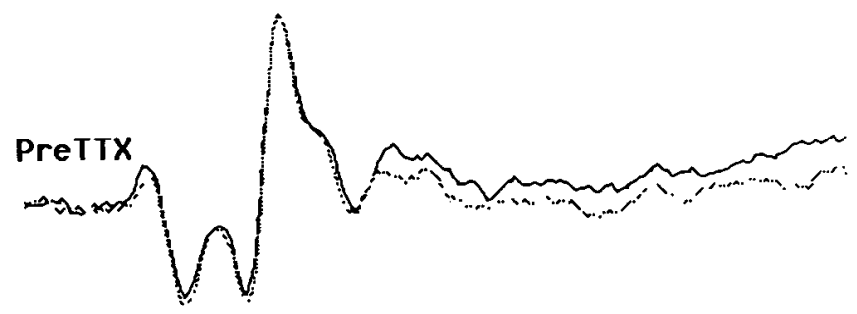

PostTTX:

$3 \mathrm{~min}$
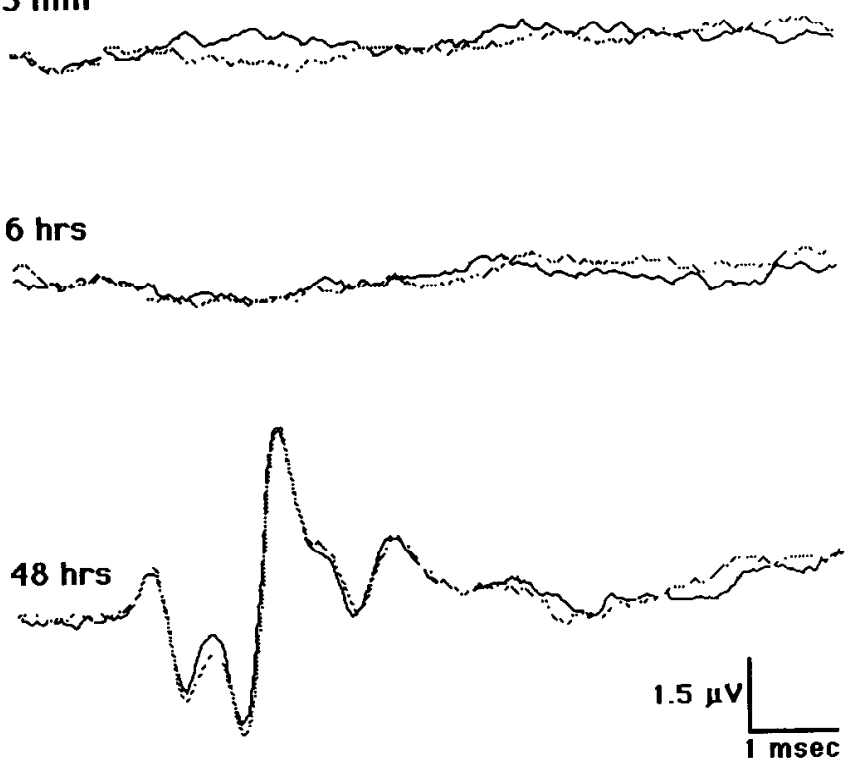

Figure 2. ABRs to $60 \mathrm{~dB}$ clicks before and after an intralabyrinthine injection of TTX embedded in PVA. In this animal, ine contralateral cochlea was removed so that elimination of ipsilaterally evoked auditory potentials results in total elimination of the ABR. Duplicate traces show reproducibility of responses.

least $3 \mathrm{hr}$ after TTX injection in all 17 surviving animals. Recovery of ABR amplitude to within $23 \%$ of pre-TTX values occurred within 2-7 d of TTX injection in 9 of 10 animals allowed to survive 1 week. In six of these nine animals, ABR amplitude returned to within $10 \%$ of its pre-TTX value. The tenth chick's ABR amplitude was still reduced by $32 \%$ from its pre-TTX value at 1 week. The remaining animals either died upon reanesthetization or were perfused prior to 1 week. Figure 3 depicts the ABR amplitude reduction and recovery for one of the animals perfused 1 week after TTX injection.

\section{GFAP immunoreactivity}

Qualitative observations. The optimal dilutions of primary antiserum for visualizing astrocytic processes in the chick brainstem were $1: 600$ to $1: 1200$ for cryostat sections and 1:4800 to $1: 7200$ for vibratome sections. In the 13 control animals, very few GFAP + processes were seen in NM, although many labeled processes were evident throughout adjacent brainstem structures. No difference in the density of GFAP + processes between the right and left NM was found in control animals. Calcium alginate-treated animals were eliminated from the control group because of the effect on ABRs. (The two calcium alginate-treated animals prepared for immunocytochemistry had more GFAP in the ipsilateral NM.) In all of the short-term TTX-treated animals, NM on the side contralateral to activity blockade had 


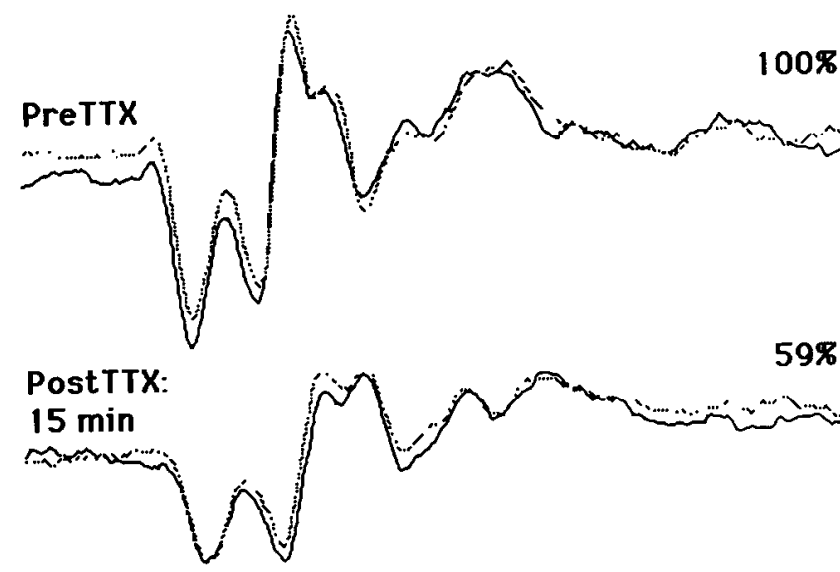

$44 \%$
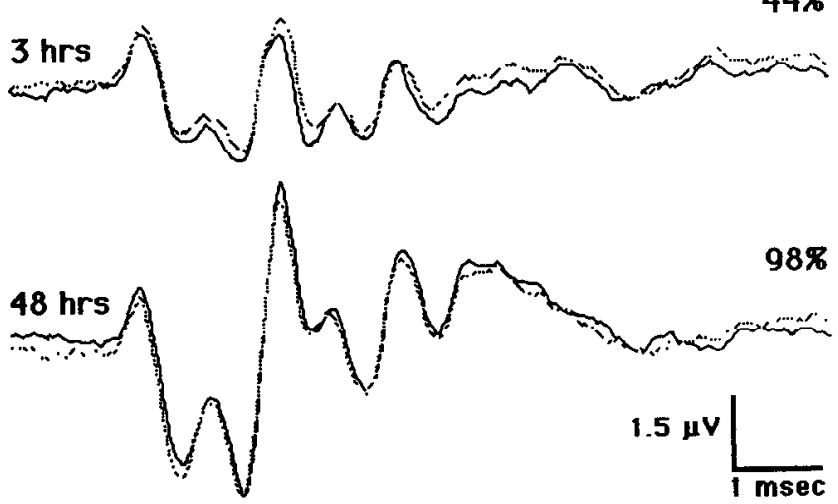

Figure 3. ABRs to $60 \mathrm{~dB}$ clicks before and after an intralabyrinthine injection of $0.3 \mathrm{~mm}$ TTX in saline. In this animal, the contralateral cochlea is intact so that elimination of ipsilaterally evoked auditory potentials results in approximately $50 \%$ reduction of ABR amplitude. Keeping the contralateral cochlea intact allows for use of the contralateral NM as a within-tissue section control for subsequent anatomical and immunocytochemical analyses.

few GFAP + processes, similar to NM in control animals. On the side ipsilateral to activity blockade, however, numerous GFAP + processes were seen both within and around NM (Fig. 4). While an increase in GFAP+ processes was apparent in NM after 1 hr of activity blockade $(n=3)$, the increase was not as dramatic as that observed after 3 or $6 \mathrm{hr}$ of activity blockade ( $n s=5,12$, respectively). The labeled processes in all of the experimental animals appeared to project into the nucleus from the NM borders. Reactive astrocytic cell bodies were rarely seen inside the nucleus.

The difference in GFAP+ processes between the control and experimental NM was not found in 9 of the 10 chicks perfused after recovery of the ABR (Fig. 5). The pattern of GFAP immunoreactivity in NM of these animals was similar to that of control animals in that few GFAP+ processes were found in either the experimental or the control NM. The one exception to this was the chick whose ABR amplitude was only $68 \%$ of normal $7 \mathrm{~d}$ after TTX injection. This chick had more GFAP+ processes in the NM ipsilateral to activity blockade.

Quantitative analysis. Quantitative results for all groups are plotted in Figure 6. The median percentage difference in area density of labeled processes in NM of control animals was $2.5 \%$ ( $n=13$; mean, 5.8\%; SEM, 5.1\%). The chicks treated with calcium alginate only were excluded from the control group because of the effect of calcium alginate on ABR amplitude.
Table 1. Neuron size and number before and after recovery from activity blockade

\begin{tabular}{llll} 
Survival time & $\begin{array}{l}\text { Number } \\
\text { of } \\
\text { animals }\end{array}$ & $\begin{array}{l}\text { Neuron size } \\
\text { (mean \% } \\
\text { difference) }\end{array}$ & $\begin{array}{l}\text { Neuron number } \\
\text { (mean \% } \\
\text { difference) }\end{array}$ \\
\hline $48 \mathrm{hr}^{a}$ & 6 & $-7.02 \pm 2.05$ & $-20.26 \pm 4.65$ \\
$7 \mathrm{~d}^{a}$ & 5 & $+1.09 \pm 1.04$ & $+2.08 \pm 0.93$ \\
$7 \mathrm{~d}$ (recovered) & 9 & $+2.32 \pm 2.33$ & $-4.56 \pm 2.00$
\end{tabular}

Neuron size and number are presented as mean \pm SEM.

a Data are from Born (1986).

The two chicks perfused $6 \mathrm{hr}$ after calcium-alginate-only treatment had a mean percentage difference in area density of GFAP+ processes of $112 \%$ (not plotted). Animals perfused $1 \mathrm{hr}$ after TTX application showed a median increase of $56 \%$ in area density of GFAP + processes in NM on the side of activity blockade ( $n=3$; mean, 66\%; SEM, 16\%). By $3 \mathrm{hr}$ of activity blockade, the median increase in GFAP + processes reached $152 \%$ ( $n=5$; mean, $178 \%$; SEM, 25\%). At $6 \mathrm{hr}$ of activity blockade, the median increase in area density of GFAP+ processes was $141 \%(n=11$; mean, $133 \%$, SEM, 34\%). The $152 \%$ median increase seen at $3 \mathrm{hr}$ and the $141 \%$ median increase found at $6 \mathrm{hr}$ are not significantly different from each other (Mann-Whitney $U, p>0.05$ ). Each of the percentage difference scores for animals perfused after 1-6 hr of activity blockade is significantly greater than the scores for control animals (MannWhitney $U, p<0.01$ ).

In contrast to the $56-152 \%$ increase in GFAP+ processes found after $1-6 \mathrm{hr}$ of unilateral activity blockade, a median difference of $3.3 \%$ was found between the experimental and control NM of the chicks allowed to recover following TTX treatment ( $n=9$; mean, $-6.0 \%$; SEM, 4\%). This difference is not significantly different from that of control animals (MannWhitney $U, p>0.05$ ). The one animal whose ABR only recovered to $68 \%$ of its pre-TTX amplitude showed a $260 \%$ increase in area density of GFAP + processes in the ipsilateral NM.

\section{Neuron size and number}

Neuron size and number did not vary as a function of recovery time, nor did changes in GFAP immunoreactivity vary with changes in neuron size or number in the recovered animals.

Changes in neuron size and number in NM were minimal in the animals allowed to recover following eighth nerve activity blockade. The chick whose ABR recovered to only $68 \%$ of its pre-TTX amplitude showed normal neuron number, but neuron size averaged $14 \%$ less in the experimental NM. The data from recovered chicks are presented in Table 1 together with similar data from Born (1986), who measured neuron size and number in NM following short- and long-term survival after injection of TTX into the perilymph of chicks. Those chicks either received TTX injections every $12 \mathrm{hr}$ and were perfused at $48 \mathrm{hr}$, or received $6 \mathrm{hr}$ of TTX injections and were perfused following a 1 week survival period during which normal activity returned. These data are presented for comparison.

\section{Discussion}

We have described proliferation of GFAP+ astrocytic processes in NM following blockade of action potentials in the afferent nerve. This astrocytic reaction occurs within the first hour of 

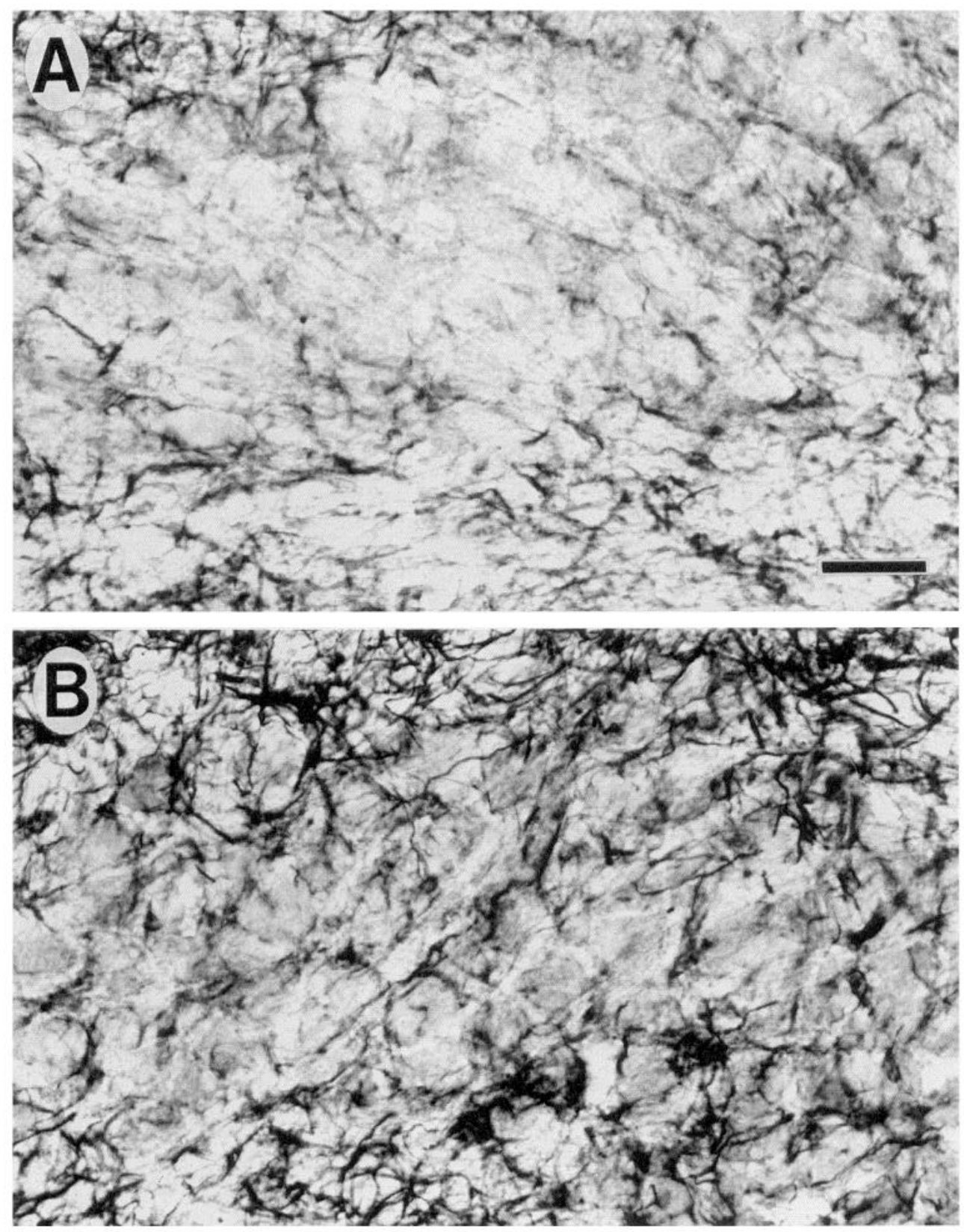

Figure 4. NM from a chick perfused $6 \mathrm{hr}$ after unilateral injection of TTX into the inner ear. The tissue section was immunolabeled with an antiserum to GFAP. A, Contralateral, control side. $B$, NM ipsilateral to activity blockade. Note increase in GFAP+ processes on the activity blockade side of the brain. Scale bar, $30 \mu \mathrm{m}$. activity blockade and plateaus by $3 \mathrm{hr}$. The increase in GFAP+ processes is sustained for as long as activity is prevented, but then disappears after neuronal activity resumes. These results indicate that the structure of astrocytic processes can be regulated by neuronal activity. Based on these results, astrocytic reactions previously described following deafferentation may have resulted, at least in part, from inactivity of local neurons rather than from neuronal degeneration alone.

This discussion will first consider the advantages and limitations of the methods employed. This will be followed by a comparison of our results to other reports of glial reactions, and to neuronal responses to similar manipulations of eighth nerve activity. Finally, we will discuss the potential functional significance of these glial changes and potential intercellular signals responsible for them.

\section{Methodological considerations}

The manipulation of NM neuronal activity in this study was achieved by exposing the peripheral processes of eighth nerve axons to TTX. Because these axons provide the only excitatory input to NM, blocking their spike activity also prevents action potentials in NM neurons. This approach allows one to block spike activity in NM without applying pharmacological agents directly to the nucleus. We have previously demonstrated that TTX injected into the perilymph does not enter the brainstem and influence CNS spike activity directly; spike activity in nuclei laminaris and angularis persists following intralabyrinthine injection of TTX (Born, 1986; Born and Rubel, 1988). Thus, we are confident that TTX infused into the perilymph did not have a direct effect on NM glial cells. 
Figure 5. NM in tissue section immunolabeled with an antiserum to GFAP from an animal perfused after recovery of the ABR. $A$, Contralateral, control side. $B$, Ipsilateral, activity blockade side. Scale bar, $30 \mu \mathrm{m}$.
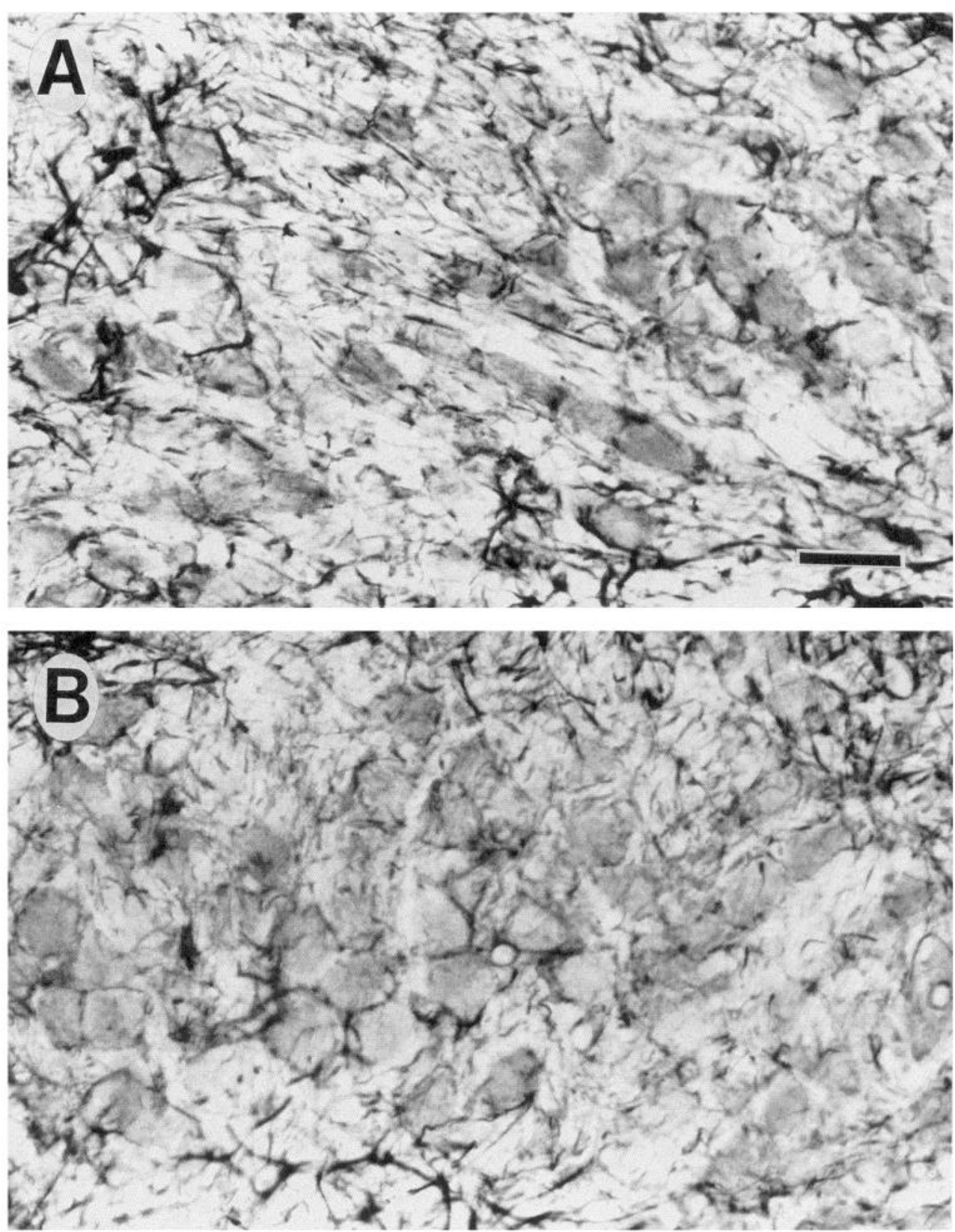

It is possible that increases in GFAP immunoreactivity might reflect an increase in available antigenic sites in existing GFAPcontaining astrocytic processes rather than growth of new processes (Eng et al., 1990). Evidence that increased GFAP immunoreactivity reflects growth of astrocytic processes rather than simply increased exposure of antigenic sites comes from the study by Rubel and MacDonald (1992). They found increases in both GFAP+ processes and silver-impregnated glial processes in NM of chicks perfused 4-6 hr after cochlea removal. Based on three-dimensional reconstructions of silver-impregnated glial cells, they found a $91 \%$ increase in glial process length and a $110 \%$ increase in the number of glial processes per cell in the NM ipsilateral to cochlea removal. We cannot exclude the possibility, however, that we observed increased GFAP immunoreactivity in astrocytic processes already present in NM. Such increased immunoreactivity could result from a change in polymerization or phosphorylation of intermediate filaments, or from the synthesis of new intermediate filaments. Since increases are also seen in NM immunoreactivity for other glial markers following cochlea removal (K. S. Canady and E. W Rubel, unpublished observations) and in light of the silverimpregnation study mentioned above, we consider the increased GFAP immunoreactivity to represent proliferation of astrocytic processes.

Increased synthesis of GFAP in NM astrocytes is not likely to occur during the short periods (1-6 hr) of activity blockade used in the present study. LePrince et al. (1991) have shown that treatment of cultured astrocytes with dibutyryl-cAMP, which induces astrocytic process elongation and increased GFAP immunoreactivity, does not stimulate increases in GFAP mRNA until $11 \mathrm{~d}$ of treatment. That increased GFAP mRNA lags behind increased GFAP immunoreactivity is consistent with our 
preliminary data on changes in GFAP mRNA in the deafferented NM (Steward, K. S. Canady, and E. R. Rubel, unpublished observations). We found that, although increases in GFAP+ processes were measurable within $1 \mathrm{hr}$ of cochlea removal, increases in GFAP mRNA were not detectable until $72 \mathrm{hr}$. By this time increased glial cell proliferation can be observed using bromodeoxyuridine labeling of newly synthesized DNA (D. Lurie, J. Shang, K. Canady, E. W Rubel, unpublished observations).

\section{Comparison to other glial reactions}

The present article is the first report of glial process proliferation in response to a documented manipulation of neuronal activity in the absence of neuropathology. It is also the first report to show reversal of glial process proliferation by restored neuronal activity. It appears that astrocytes can react to a wide variety of changes in the neural environment (Lindsay, 1986; Malhotra et al., 1990). While all of these reactions may involve an increase in GFAP expression, there may be important differences in the nature and purpose of the reactions. Perhaps there exists a continuum of severity and complexity of glial reactions with the reversible astrocytic response to neuronal inactivity representing a simple and mild reaction. Likewise, the reaction to a stab wound, in which the blood-brain barrier has been disrupted and axons have been severed, might represent a rather severe and complex reaction involving multiple signals and other cell types (e.g., macrophages). Intermediate to these two examples would be the glial response to deafferentation that eventually results in the degeneration of afferent terminals and, in some systems, atrophy or death of the postsynaptic neurons (Rubel et al., 1990).

The report of increased GFAP+ process in NM following cochlea removal (Rubel and MacDonald, 1992) may reflect a two-stage glial reaction to deafferentation. The increase in GFAP+ processes ranged from approximately $50 \%$ to $100 \%$ at 1-24 hr after cochlea removal, but exceeded $500 \%$ after $72 \mathrm{hr}$. The more dramatic reaction observed at $72 \mathrm{hr}$ may reflect an astrocytic reaction to the degeneration of afferent nerve terminals and some of the postsynaptic neurons. The early phase of this reaction, however, is likely due to the loss of eighth nerve activity and differs from that described for other glial reactions to deafferentation. For example, Anders and Johnson (1990) found no increase in GFAP+ processes prior to $48 \mathrm{hr}$ following deafferentation of the piriform cortex in rats. Thus, glial responses to similar manipulations may differ between neural systems, perhaps as a function of differences in the impact of deafferentation on neuronal activity. Alternatively, differing reports may reflect differing criteria for increased GFAP immunoreactivity or limitations of comparisons between animals.

In other models of reactive gliosis, decreases in GFAP immunoreactivity have been reported at early (12-24 hr) survival times [Eriksdotter-Nilsson et al., 1987 (excitotoxic lesion); Miyake et al., 1988 (stab wound)]. Increases in GFAP immunoreactivity first appeared at $48-96 \mathrm{hr}$ in these two examples of neural trauma. Consideration of the variety of glial reactions and their etiologies becomes more important in light of these examples.

The only previous reports of reversal of an astrocytic reaction (increased GFAP+ processes) involved neural systems with a capacity to regenerate. Anders and Johnson (1990) described increased GFAP labeling in the rat olfactory bulb and piriform cortex following transection of the olfactory nerve. At 1 month after transection, sufficient time for the nerve to regenerate,

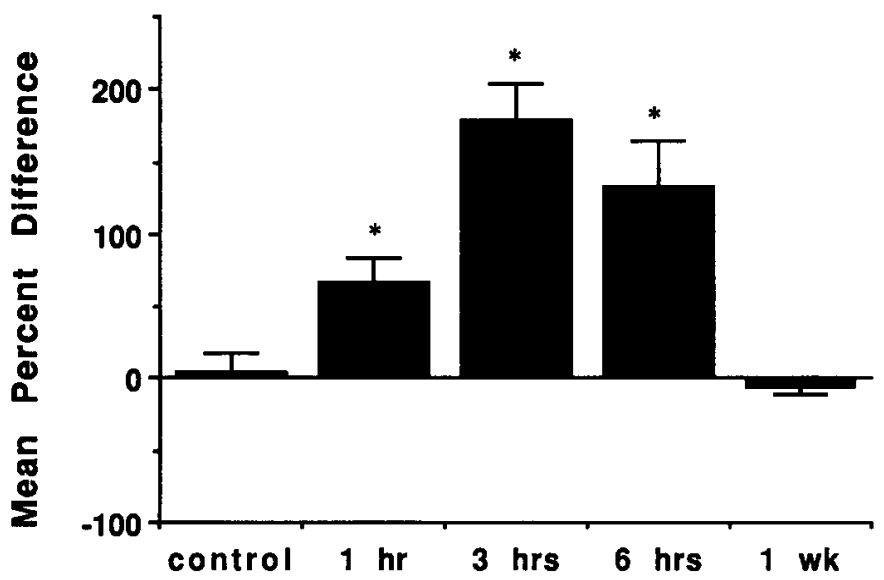

Figure 6. Mean percentage difference between ipsilateral and contralateral NM in percentage of area occupied by GFAP+ processes for animals perfused at various survival times following intralabryinthine TTX injection. Positive values reflect increased GFAP immunoreactivity in NM ipsilateral to TTX injection. Error bars represent SEM. Asterisks indicate groups that differ significantly from controls (MannWhitney $U, p<0.01$ ).

GFAP immunoreactivity in the piriform cortex returned to control levels. Similarly, Stafford et al. (1990) reported the appearance of GFAP + processes in goldfish optic nerve following nerve crush. No GFAP labeling was found after the nerve had regenerated. Our results raise the possibility that, in these regenerating systems, the astrocytic reactions are reversed by the resumption of normal levels of neuronal activity.

One example of the lability of astrocytic processes in vivo in the absence of neuropathology comes from studies of the supraoptic nucleus of the rat (Hatton and Tweedle, 1982; Salm et al., 1985). Using both immunocytochemistry for GFAP at the light microscopic level and ultrastructural analysis, these investigators have described the lactation-associated withdrawal of astrocytic processes normally interposed between magnocellular neurons. In lactating rats, the retraction of astrocytic processes is associated with reduced immunoreactivity for GFAP in the supraoptic nucleus. These glial changes may also be regulated by changes in neuronal activity.

\section{Relation to neuronal changes}

Previous work from our laboratory concerning the influence of eighth nerve activity on neurons in NM has been summarized in a recent review (Rubel et al., 1990). In brief, neuron size and number are reduced in the ipsilateral NM following cochlea removal or intralabyrinthine injection of TTX. After $48 \mathrm{hr}$ of eighth nerve blockade by TTX, NM neuron size is reduced and $20-30 \%$ of the neurons are lost. As early as 1-6 hr after TTX application, neuron size is normal but neuronal metabolism is altered. For example, protein synthesis is reduced by $40 \%$ in the ipsilateral NM $1 \mathrm{hr}$ after TTX injection. Thus, the glial reaction, which is evident within $1 \mathrm{hr}$ of TTX injection, cannot be "filling empty space left by dying neurons" (Björklund et al., 1986). Although some neurons may be showing early signs of dying, they have not yet created empty space. The potential significance of changes in neuronal metabolism concomitant with a glial reaction is curious. It would be interesting, though perhaps difficult, to learn if the neuronal change precipitates the glial rcaction or vicc versa.

In the animals allowed to recover following activity blockade, 
neuronal size and number were not significantly altered (see also Born, 1986). These neuronal measures were included in the study as a second index of recovery from activity blockade. All chicks whose ABRs recovered to $77 \%$ or more of the pre-TTX amplitude recovered normal neuron size and number as well as normal levels of GFAP immunoreactivity. The one chick whose ABR recovered to only $68 \%$ of its pre-TTX amplitude recovered normal neuron number, but neuron size averaged $14 \%$ less and area density of GFAP + processes averaged $260 \%$ greater in the experimental NM.

\section{Functional significance}

The functional significance of increases in GFAP+ processes in NM is unknown. It may be that newly extended processes disrupt synaptic contacts onto NM neurons (Meshul et al., 1987; Pomeroy and Purves, 1988). Most forms of neural modification due to experience involve a chronic change in activity through one or more neural circuits. The ensuing modification in neural function is upregulation or downregulation of synaptic efficacy in one or more circuits. An intriguing possibility suggested by the present results is that activity-induced modulation of astrocytic branching patterns influences synaptic efficacy by regulating the spatial summation of synaptic inputs. The fact that statistically reliable increases in glial processes can be seen at the light microscopic level after only $1 \mathrm{hr}$ of activity deprivation suggests that glial processes that surround most synaptic complexes in the CNS may play a major role in regulating the number of active sites between synaptic partners. Incorporation of glial changes that regulate synaptic efficacy in models of learning and memory would be relatively simple, and one can imagine several ways to evaluate this hypothesis by anatomical or physiological studies in more traditionally used preparations for examining synaptic plasticity.

Alternatively, the astrocytic reaction may reflect an attempt by the sparse glial population of NM to increase its ability to supply nutrients or clear wastes from metabolically challenged neurons. Another possibility is that the reactive glial processes create borders between those neurons destined to die and those that will survive. Most descriptions of glial reactions are of sites of severe neural trauma such as stab wounds and axotomized nuclei (Adrian and Williams, 1973; Graeber and Kreutzberg, 1988; Janeczko, 1988; Topp et al., 1989). In these examples, astrocytes appear to form a scar around the lesion. Speculations on the roles of reactive glial cells based on these findings often include phagocytosis of debris (Varon and Somjen, 1979), providing a physical barrier to the spread of damage (Reier, 1986), and replacement of space once occupied by neurons (Björklund et al., 1986). It seems unlikely that these roles would apply to the reactive glial processes in the temporarily silenced NM since severe damage has not occurred.

Some investigators have suggested that reactive astrocytes inhibit neuronal regeneration following axotomy (Reier, 1986). It is also unlikely that this principle would apply to all systems and to all conditions in which increased GFAP immunoreactivity is found, since in the rat olfactory system, as mentioned above, increased GFAP immunoreactivity occurs prior to regeneration (Anders and Johnson, 1990). Reier (1986) himself cites several examples that challenge the hypothesis that gliosis prevents axonal regeneration. For systems in which reactive astrocytes do appear to impede neuronal regeneration, it may be worthwhile to explore the influence neuronal activity could have on the astrocytic reaction. Such a model might create an opportunity to test the hypothesis that reactive astrocytes impede regeneration.

\section{Potential signals}

We have recently begun to explore potential signals by which neuronal activity regulates astrocytic processes using an in vitro slice preparation containing the brainstem auditory nuclei ( $\mathrm{Hy}$ son and Rubel, 1989). Results from these studies support the hypothesis that ionic fluxes associated with neuronal depolarization may be involved (Hyson et al., 1990). For example, extracellular potassium $\left(\mathrm{K}^{+}\right)$rises from a concentration of 3 $\mathrm{mM}$ to approximately 5-10 $\mathrm{mm}$ with neuronal activity (Somjen, 1979) and elevated $\mathrm{K}^{+}{ }_{\circ}$ may inhibit astrocytic processes. This is supported by our previous study in which the GFAP expression of cultured astrocytes was found to be inversely related to $\mathrm{K}^{+}$(Canady et al., 1990).

\section{References}

Adrian EK Jr, Williams MG (1973) Cell proliferation in injured spinal cord. J Comp Neurol 151:1-24.

Amaducci L, Forno KI, Eng LF (1981) Glial fibrillary acidic protein in cryogenic lesions of the rat brain. Neurosci Lett 21:27-32.

Anders JJ, Johnson JA (1990) Transection of the rat olfactory nerve increases glial fibrillary acidic protein immunoreactivity from the olfactory bulb to the piriform cortex. Glia 3:17-25.

Björklund H, Olson L, Dahl D, Scwarcz R (1986) Short- and longterm consequences of intracranial injections of the excitotoxin, quinolinic acid, as evidenced by GFA immunohistochemistry of astrocytes. Brain Res 371:267-277.

Born DE (1986) The role of neuronal activity in regulating the structure and function of auditory neurons. $\mathrm{PhD}$ thesis, University of Virginia.

Born DE, Rubel EW (1985) Afferent influences on brain stem auditory nuclei of the chicken: neuron number and size following cochlea removal. J Comp Neurol 231:435-445.

Born DE, Rubel EW (1988) Afferent influences on brain stem auditory nuclei of the chicken: presynaptic action potentials regulate protein synthesis in nucleus magnocellularis. J Neurosci 8:901-919.

Born DE, Durham D, Rubel EW (1991) Afferent influences on brainstem auditory nuclei of the chick: nucleus magnocellularis neuronal activity following cochlea removal. Brain Res 557:37-47.

Canady KS, Ali-Osman F, Rubel EW (1990) Extracellular potassium influences DNA and protein syntheses and glial fibrillary acidic protein expression in cultured glial cells. Glia 3:368-374.

Cass SP, Goshgarian HG (1990) Increased glial fibrillary acidic protein immunoreactivity in astrocytes within the lateral vestibular nucleus of the cat folloiwng labyrinthectomy and vestibular neurectomy. Ann Otol Rhinol Laryngol 99:221-227.

Eng LF, DeArmond SJ (1982) Immunocytochemical studies of astrocytes in normal development and disease. Adv Cell Neurobiol 3:145171.

Eng LF, D'Amelio FE, Smith ME (1990) Dissociation of GFAP intermediate filaments in EAE: observations in the lumbar spinal cord. Glia 2:308-317.

Eriksdotter-Nilsson M, Björklund H, Dahl D, Olson L, Ingvar M (1987) Sustained seizures cause circumscribed cerebral changes in glial fibrillary acidic protein, neurofilament and laminin immunofluorescence. Exp Brain Res 69:155-166.

Graeber MB, Kreutzberg GW (1988) Delayed astrocyte reaction following facial nerve axotomy. J Neurocytol 17:209-220.

Hatton GI, Tweedle CD (1982) Magnocellular neuropeptidergic neurons in hypothalamus: increases in membrane apposition and number of specialized synapses from pregnancy to lactation. Brain Res Bull 8:197-204.

Hyson RL, Rubel EW (1989) Transneuronal regulation of protein synthesis in the brain stem auditory system of the chick requires synaptic activation. J Neurosci 9:2835-2845.

Hyson RL, Canady KS, Rubel EW (1990) Neuronal activity regulates astrocytic processes in a brain slice preparation of the avian auditory system. Soc Neurosci Abstr 16:828.

Janeczko K (1988) The proliferative response of astrocytes to injury in neonatal rat brain. A combined immunocytochemical and autoradiographic study. Brain Res 456:280-285. 
LePrince G, Fages C, Rolland B, Nunez J, Tardy M (1991) DBcAMP effect on the expression of GFAP and of its encoding mRNA in astroglial primary cultures. Glia 4:322-326.

Lindsay RM (1986) Reactive gliosis. In: Astrocytes (Federoff S, Vernadakis A, eds.), pp 231-262. Orlando, FL: Academic.

Malhotra SK, Shnitka JK, Elbrink J (1990) Reactive astrocytes-a review. Cytobios 61:133-160.

Meshul CK, Frederick JS, Herndon RM (1987) Astrocytes play a role in regulation of synaptic density. Brain Res 402:139-145.

Miyake T, Hattori T, Fukuda M, Kitamura T, Fujita S (1988) Quantitative studies on proliferative changes of reactive astrocytes in mouse cerebral cortex. Brain Res 451:133-138.

Parks TN (1981) Morphology of axosomatic endings in the avian cochlear nucleus: nucleus magnocellularis of the chicken. J Comp Neurol 203:425-440.

Parks TN, Rubel EW (1978) Organization and development of brain stem auditory nuclei of the chicken: primary afferent projections. J Comp Neurol 180:439-448.

Pomeroy SL, Purves D (1988) Neuron/glia relationships observed over intervals of several months in living mice. J Cell Biol 107:11671175.

Reier PJ (1986) Gliosis following CNS injury: the anatomy of astrocytic scars and their influences on axonal elongation. In: Astrocytes (Federoff S, Vernadakis A, eds), pp 263-324. Orlando, FL: Academic
Rubel EW, MacDonald GH (1992) Rapid growth of astrocytic processes in $\mathbf{n}$. magnocellularis following cochlea removal. J Comp Neurol, in press.

Rubel EW, Hyson RL, Durham D (1990) Afferent regulation of neurons in the brain stem auditory system. J Neurobiol 21:169-196.

Salm AK, Smithson KG, Hatton GI (1985) Lactation-associated redistribution of the glial fibrillary acidic protein within the supraoptic nucleus. Cell Tissue Res 242:9-15.

Somjen GG (1979) Extracellular potassium in the mammalian central nervous system. Annu Rev Physiol 41:159-177.

Stafford DA, Shehab SAS, Nona SN, Cronly-Dillon JR (1990) Expression of glial fibrillary acidic protein (GFAP) in goldfish optic nerve following injury. Glia 3:33-42.

Topp KS, Faddis BT, Vijayan VK (1989) Trauma-induced proliferation of astrocytes in the brains of young and aged rats. Glia 2:201211.

Varon SS, Somjen GG (1979) Neuron-glia interactions. Neurosci Res Prog Bull 17:1-187

Wong-Riley MTT (1972) Terminal degeneration and glial reactions in the lateral geniculate nucleus of the squirrel monkey after eye removal. J Comp Neurol 144:61-92. 